\title{
How Malaria Parasites Acquire Nutrients From Their Host
}

\author{
Natalie A. Counihan, Joyanta K. Modak and Tania F. de Koning-Ward* \\ School of Medicine, Deakin University, Waurn Ponds, VIC, Australia
}

Plasmodium parasites responsible for the disease malaria reside within erythrocytes. Inside this niche host cell, parasites internalize and digest host hemoglobin to source amino acids required for protein production. However, hemoglobin does not contain isoleucine, an amino acid essential for Plasmodium growth, and the parasite cannot synthesize it de novo. The parasite is also more metabolically active than its host cell, and the rate at which some nutrients are consumed exceeds the rate at which they can be taken up by erythrocyte transporters. To overcome these constraints, Plasmodium parasites increase the permeability of the erythrocyte membrane to isoleucine and other low-molecular-weight solutes it requires for growth by forming new permeation pathways (NPPs). In addition to the erythrocyte membrane, host nutrients also need

OPEN ACCESS

Edited by:

Carmen Faso,

University of Bern, Switzerland

Reviewed by:

Volker Theo Heussler,

University of Bern, Switzerland

Curtis Okamoto,

University of Southern California,

Los Angeles, United States

*Correspondence:

Tania F. de Koning-Ward

taniad@deakin.edu.au

Specialty section:

This article was submitted to

Membrane Traffic,

a section of the journal

Frontiers in Cell and Developmental

Biology

Received: 04 January 2021

Accepted: 24 February 2021

Published: 25 March 2021

Citation:

Counihan NA, Modak JK and de Koning-Ward TF (2021) How Malaria Parasites Acquire Nutrients

From Their Host.

Front. Cell Dev. Biol. 9:649184. doi: 10.3389/fcell.2021.649184 to cross the encasing parasitophorous vacuole membrane (PVM) and the parasite plasma membrane to access the parasite. This review outlines recent advances that have been made in identifying the molecular constituents of the NPPs, the PVM nutrient channel, and the endocytic apparatus that transports host hemoglobin and identifies key knowledge gaps that remain. Importantly, blocking the ability of Plasmodium to source essential nutrients is lethal to the parasite, and thus, components of these key pathways represent potential antimalaria drug targets.

Keywords: Plasmodium, malaria, nutrients, new permeation pathway, transporters

\section{INTRODUCTION}

Malaria is a devastating infectious disease caused by protozoan parasites belonging to the species Plasmodium, with an estimated 228 million cases in 2018 alone (World Health Organization, 2019). Despite a modest reduction in the burden of malaria in the last 20 years, it remains a global health problem because of the absence of an effective vaccine and limited chemotherapeutic options as a result of drug resistance. The most severe form of malaria is caused by Plasmodium falciparum that is transmitted between people via a mosquito vector (Figure 1a). After infection, the parasite has a complex lifecycle that includes an asymptomatic liver stage (Figure 1b). Following replication inside hepatocytes, merozoites are released into the blood where they invade erythrocytes to initiate the blood stage of the parasite lifecycle; here, rapid asexual replication occurs, and the clinical symptoms of malaria become evident (Figure 1c).

Plasmodium falciparum is an obligate intracellular parasite and is dependent on its host to supply the nutrients required to support its development. However, P. falciparum faces some challenges by selecting to reside in mature, metabolically inactive erythrocytes. While the parasites are able to take up hemoglobin from the host cell cytoplasm and rapidly metabolize it, human hemoglobin does not contain all the amino acids necessary for P. falciparum growth; isoleucine is absent, and 


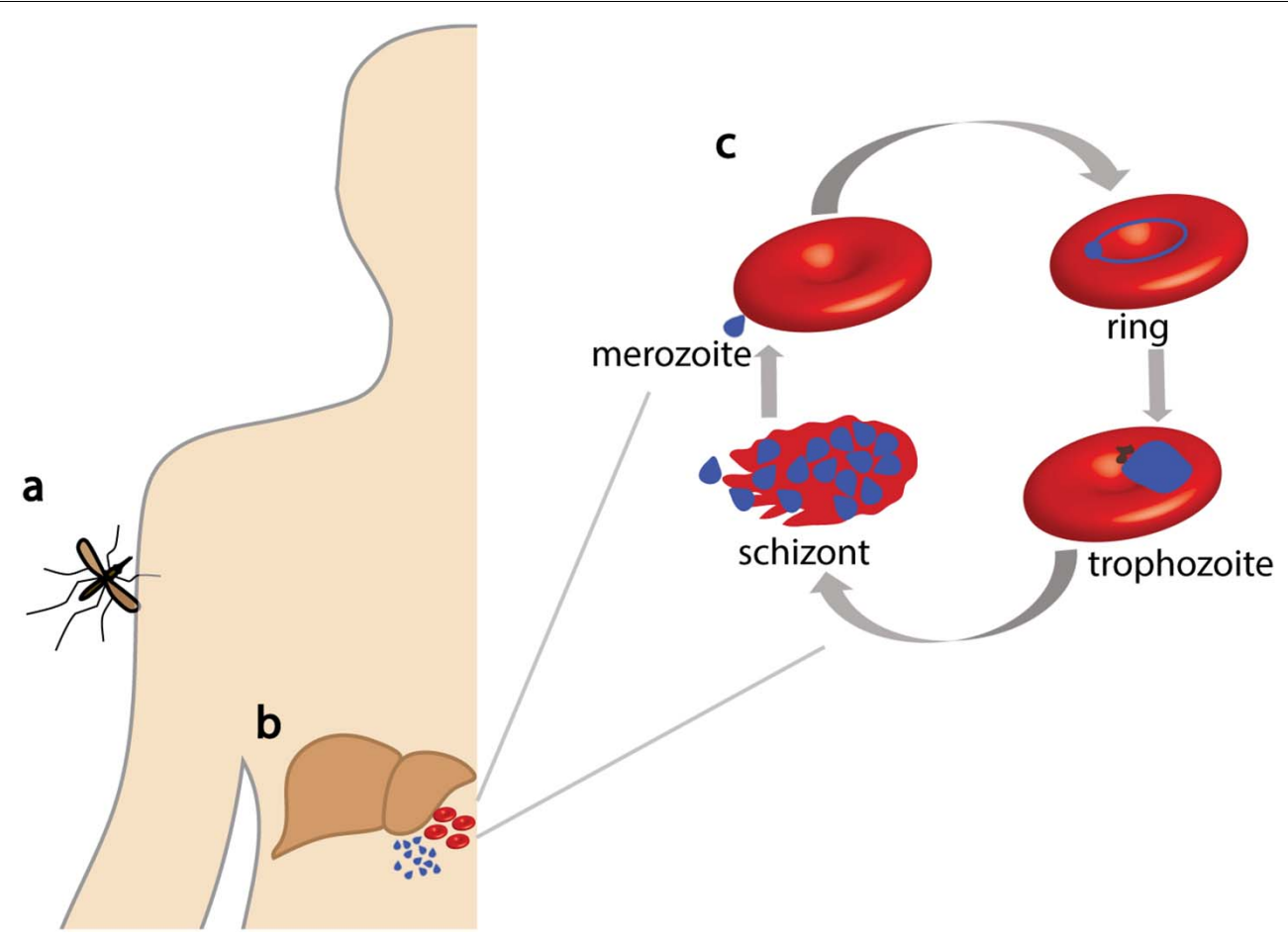

FIGURE 1 | Lifecycle of Plasmodium falciparum. P. falciparum parasites are injected into a human host via mosquito bites (a) where they then travel to the liver (b). After parasite replication, merozoites are released into the blood where they invade erythrocytes to commence the asexual replication cycle (c). Parasites develop within the erythrocyte in distinct forms (ring, trophozoite, and schizont) until erythrocyte lysis occurs, releasing merozoites for reinvasion.

methionine, for example, is poorly represented, and thus these amino acids must be acquired from the human serum (Sherman, 1977; Divo et al., 1985; Spry et al., 2010). So too does pantothenate (vitamin $B_{5}$ ), the precursor to coenzyme $A$, as it cannot be synthesized by the parasite de novo, and it is also essential for parasite growth (Saliba et al., 1998). Although $P$. falciparum can synthesize thiamine (vitamin $\mathrm{B}_{1}$ ), it cannot do so in sufficient quantities (Wrenger et al., 2006). Also, rate-limiting to parasite growth are the diminishing intracellular pools of other nutrients including folate and purines (Liu et al., 2006; Wang et al., 2007), and as P. falciparum is unable to synthesize purines, it is dependent upon the salvage of exogenous purines via the purine salvage pathway (Reyes et al., 1982; Downie et al., 2008). While $P$. falciparum has some capacity to synthesize its own lipids (reviewed in O'Neal et al., 2020), several classes of lipids are ratelimiting and must also be obtained from an extracellular source (Gulati et al., 2015; O’Neal et al., 2020).

As $P$. falciparum resides within a parasitophorous vacuole (PV) in the host erythrocyte (Figure 2), nutrients acquired from the extracellular milieu must traverse otherwise impermeable membranes, namely, the erythrocyte plasma membrane (EPM), the PV membrane (PVM), and the parasite plasma membrane (PPM). The erythrocyte plasma membrane harbors a range of specialized transporters that form channels, carriers, or pumps to facilitate the passage of substrates. However, not all substrates required by Plasmodium (e.g., isoleucine and pantothenate) can be obtained via the endogenous transporters, or they are transported in insufficient quantity (Saliba et al., 1998; Martin and Kirk, 2007). Therefore, additional mechanisms must be employed to enable $P$. falciparum to acquire essential nutrients from the host serum. This is achieved through the creation of new permeation pathways (NPPs) that facilitate the transport of a broad range of substrates across the erythrocyte membrane (Ginsburg et al., 1985; Desai et al., 1993). NPPs result from modifications to the host erythrocyte membrane (Figure 2); they behave as channels and display a preference for anions over cations (Kirk et al., 1994), but also likely act to dispel waste products generated from hemoglobin digestion and metabolic processes and to maintain ion gradients essential for cell function. NPPs are established at the erythrocyte membrane from $15 \mathrm{~h}$ after invasion and reach a plateau after $36 \mathrm{~h}$ (Staines et al., 2001). Despite the importance of this channel to parasite survival and its potential as a drug target, until recently, very little was known about its molecular composition, especially compared with our current knowledge of other P. falciparum transporters (reviewed in Martin, 2020). The molecular makeup of the "sieve" that allows host nutrients to then cross the encasing PVM so that they can then be transported by the many transporters that decorate the PPM has also only just been revealed. So too have components of the endocytic apparatus that traffics hemoglobin to the digestive food vacuole. This review discusses the strategies the parasite employs to obtain nutrients from the host serum and host cell cytoplasm and the recent advances that have been made in identifying the molecular constituents of these pathways. 


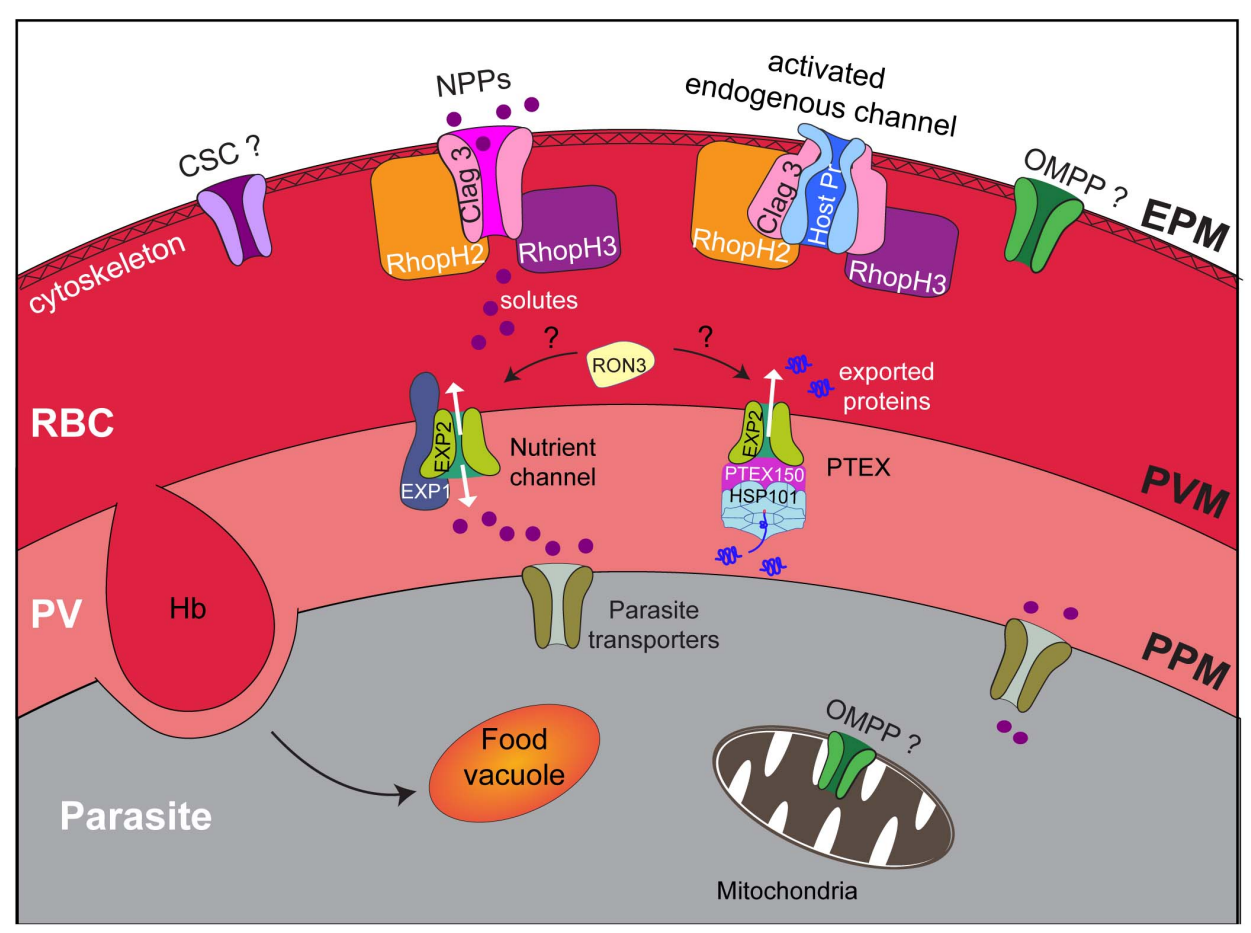

FIGURE 2 | Nutrient acquisition by P. falciparum relies on membrane transporters and the cytostome. Upon infection by Plasmodium parasites, erythrocytes are extensively remodeled to accommodate the growing parasite. The parasite, contained by its own PPM, is encased within a parasitophorous vacuole (PV) and a PV membrane (PVM). Each membrane in the infected erythrocyte, including the erythrocyte plasma membrane (EPM), is modified to include transporters that enable the parasite to access nutrients from its host. These include the NPPs at the EPM, the nutrient channel at the PVM, and transporters at the PPM, whereas hemoglobin uptake and transport to the food vacuole occur via the cytostome. The NPP channel is proposed to be a CLAG3 dimer/oligomer that associates with RhopH2 and RhopH3. Alternatively, CLAG3, RhopH2, and RhopH3 may activate an endogenous host channel. The contribution of other parasite-encoded channels to the NPPs such as the calcium-dependent, stress-gated ion channel (CSC), and OMPP is unknown; the localization of both of these proteins is yet to be ascertained. The nutrient channel at the PVM comprises EXP2, with EXP1 being critical for proper distribution of the EXP2 nutrient channel. EXP2 also forms the pore of the translocon of exported proteins (PTEX). RON3 is implicated in both nutrient uptake across the PVM and protein export with an unknown function. The PPM is also decorated with a raft of transporters that facilitate the uptake of diverse substrates to satisfy the metabolic requirements of the growing parasite.

\section{CROSSING THE ERYTHROCYTE MEMBRANE}

The human erythrocyte membrane contains more than 50 types of transmembrane proteins in its lipid bilayer that are responsible for a range of functions including substrate transport, cell adhesion, and structural integrity (reviewed in Pretini et al., 2019). Substrate transport is facilitated by ATPpowered transporters, organic cation and anion channels, glucose transporters, $P$-type ATPases, urea transporters, and aquaporins, as well as monocarboxylate transporter 1 (MCP1), anionexchanger 1, and equilibrative nucleoside transporter 1 (Pretini et al., 2019). These transporters serve to maintain the selective permeability of the membrane. It has long been recognized that $P$. falciparum infection induces a measurable increase in the permeability of host erythrocytes (Overman, 1947) to a diverse range of structurally unrelated low molecular mass solutes including sugars, amino acids, vitamins, purines, and ions (reviewed in Martin, 2020), and this change is attributed to NPPs (Ginsburg et al., 1985; Kirk et al., 1994). As the NPPs have the characteristics of an anion-selective channel, it is referred to as the Plasmodium surface anion channel (PSAC) (reviewed in
Desai, 2012). Despite the recognition of the existence of NPPs for several decades, its biochemical composition has not been well defined, and indeed, it is not known whether PSAC represents the only NPP and whether the NPPs are solely parasite-derived or comprise host cell constituents.

\section{Contribution of CLAG Proteins to NPPs}

The $P$. falciparum gene products encoded by clag3.1 (PF3D7_0302500) and clag3.2 (PF3D7_0302200) genes were the first parasite-encoded proteins implicated in NPPs and were identified using nutrient restriction, NPP inhibitors, and genetic approaches (Nguitragool et al., 2011; Pillai et al., 2012; Figure 2). Parasites in which CLAG3 expression has been depleted through either epigenetic silencing or in vitro selection exhibit delayed in vitro growth (Comeaux et al., 2011) and altered PSAC activity (Mira-Martinez et al., 2013; Sharma et al., 2013, 2015); yet, interestingly, parasites are still able to survive. Even complete knockout of the clag3 genes is not lethal, and transport activity is not completely lost in these parasites (Gupta et al., 2020). In the latter case, parasites only displayed a fitness cost when grown in a modified medium that more closely resembles human plasma. 
That the CLAG3 proteins were implicated in NPPs was surprising as they do not resemble known transporters, and moreover, it was expected that proteins comprising the NPP channel would be essential for parasite survival given the vital nature of the substrates it transports. However, clag3.1 and clag3.2 belong to a unique Plasmodium multigene family referred to as RhopH1, which also comprises clag2, clag8, and clag9 in P. falciparum (Kaneko et al., 2001; Ling et al., 2004; Kaneko et al., 2005). As all five clag genes have the same intron-exon structure and are highly conserved, it is conceivable that the loss of the clag3 genes can be functionally complemented by the other paralogs. Unfortunately, this is very difficult to assess in $P$. falciparum given the technical limitations of knocking out multiple clag paralogs in the one parasite line. Alternatively, it is possible that the different clag paralogs form distinct channels that facilitate the uptake of different solutes. To date, gene knockouts of clag2 and clag8 have not been reported in the literature to assess this. However, clag2 expression was found to be silenced in blasticidin-resistant parasites in which silencing of both clag3 genes led to reduced uptake of blasticidin (Sharma et al., 2013, 2015). This is despite clag2 expression being independent of the epigenetically regulated clag3 switching program (Cortés et al., 2007). This finding suggests that clag2 may also contribute to NPP function. For $\operatorname{clag} 9$, several studies have shown that this gene is not essential for P. falciparum growth in vitro. Interestingly, CLAG9 is the most distinct of all the CLAG paralogs and lacks the small hypervariable region that is present in its paralogs and which has been shown to be exposed at the erythrocyte surface (Nguitragool et al., 2011, 2014). However, to date, no studies have directly examined the impact of the loss of clag9 on solute uptake. Prior studies have instead linked CLAG9 to cytoadherence of infected erythrocytes to CS32 melanoma cells and the endothelial receptor CD36, potentially via assisting with the trafficking of the major virulence factor PfEMP1 onto the erythrocyte surface (Trenholme et al., 2000; Goel et al., 2010). However, a subsequent study observed that the loss of binding of infected erythrocytes to CD36 was a result of expression of a non-functional PfEMP1 rather than lack of clag9 (Nacer et al., 2011). CLAG9 has also been linked to merozoite binding to erythrocytes by interacting with the glycophorin A-band3 receptor-co-receptor complex (Chourasia et al., 2020). Although it is not uncommon for protein and protein fragments to bind non-specifically to erythrocyte membranes, $\mathrm{RhopH} 3$ that forms a complex with clag9 (discussed below) has also been shown to be involved in erythrocyte invasion. Nevertheless, at this stage, it still remains to be formally examined whether CLAG9 also plays a role in NPP activity.

\section{Contribution of RhopH2 and RhopH3 to NPP}

The CLAG3 proteins are synthesized toward the end of the parasite asexual cell cycle, where they are stored in the rhoptry organelle in a complex, termed the RhopH complex, with two unrelated proteins named RhopH2 and RhopH3, which are encoded by single copy genes [(Cooper et al., 1988) and reviewed in Counihan et al. (2013)]. The RhopH protein complex is highly conserved among Plasmodium species, although the number of Clag genes varies among Plasmodium species. Upon merozoite invasion, all three proteins are transferred to the newly infected erythrocyte (Vincensini et al., 2008) and are transported to the host cell membrane via undefined trafficking routes. At the erythrocyte membrane, RhopH1, RhopH2, and RhopH3 exist as a complex (Figure 2; Sam-Yellowe et al., 1988) in a stoichiometry yet to be resolved, where $\mathrm{RhopH} 2$ has been shown to associate with host skeletal proteins (Counihan et al., 2017). Both the rhopH2 and rhopH3 genes are refractory to deletion and essential for Plasmodium survival (Cowman et al., 2000; Janse et al., 2011; Zhang et al., 2018). Using inducible knockdown/knockout approaches, depletion of RhopH2 and RhopH3 caused a significant defect in parasite growth, and NPP activity was limited (Counihan et al., 2017; Ito et al., 2017; Sherling et al., 2017). Interestingly, depletion of RhopH2 resulted in a reduction of essential vitamins and cofactors including folate and decreased de novo synthesis of pyrimidines, which likely contributed to the delayed growth phenotype observed (Counihan et al., 2017). Normally, P. falciparum requires $\sim 48 \mathrm{~h}$ to complete a lifecycle, and rapid growth and maturation are observed in the second half of the cycle (Figure 1); reduced levels of $\mathrm{RhopH} 2$ and $\mathrm{RhopH} 3$ resulted in parasites stalling about midway through their lifecycle, which is consistent with when NPPs are active (15-36 $\mathrm{h}$ after invasion).

\section{What Is the Channel-Forming Component of the NPP?}

There is compelling evidence that the $\mathrm{RhopH}$ proteins participate in NPP formation, but there are still fundamental questions concerning the makeup of NPP that need to be determined, particularly the component(s) that form the actual channel through the erythrocyte membrane. The CLAG proteins do not resemble any known proteins, including known transporters, although some of the top hits to the CLAG3 proteins using the protein prediction and structure program iTasser (Zhang, 2008) are channel proteins. The CLAGs are predicted to harbor a region that may form a transmembrane domain that is lined with polar residues on one side and non-polar on the other, reminiscent of the domains that form water-filled pores (Sharma et al., 2015), and a pool of CLAG3 is integral to the membrane (Nguitragool et al., 2011).

A soluble form of RhopH protein complex structure has recently been solved via cryoelectron microscopy (Schureck et al., 2021). Structural analysis reveals a heterotrimeric complex of CLAG3, RhopH2, and RhopH3 with a 1:1:1 stoichiometry. CLAG3 is situated in the middle of the complex, where it interacts tightly with RhopH2 and RhopH3. However, the predicted transmembrane domain of CLAG3, which does indeed form an a-helix near the CLAG3 C-terminus, is buried in the core of the RhopH complex and is not exposed to solvent. So too is the hypervariable region that was previously shown to be exposed on the surface of infected erythrocytes (Nguitragool et al., 2011). Thus, in order for the complex to be incorporated into the membrane, large-scale conformational changes would be required. The process(es) that may drive this are unknown 
but could potentially involve posttranslational modifications such as phosphorylation or regulation of allosteric disulfides or may require interactions with proteins or lipids at the erythrocyte plasma membrane.

RhopH2 and RhopH3 are even less convincing as potential channel proteins, showing no structural homology to known channel proteins. Moreover, protease protection assays indicate that $\mathrm{RhopH} 2$ and $\mathrm{RhopH} 3$ are not exposed on the erythrocyte surface (Ito et al., 2017). Some protein prediction programs such as Phobius suggest the presence of at least 1 transmembrane domain in RhopH2 and RhopH3, and thus, there is the possibility that CLAG forms a functional channel with RhopH2 and RhopH3 or indeed forms homo-oligomers or oligomerizes with other proteins to form a channel (see Figure 2 for a model). However, the predicted transmembrane domains of RhopH2 and RhopH3 are also buried in the RhopH soluble structure, 46$101 \AA$ apart from the CLAG3 predicted transmembrane domain. Thus, massive rearrangement of the complex structure would be required if the RhopH2, RhopH3, and CLAG3 transmembrane domains come together to form a channel in the erythrocyte plasma membrane (Schureck et al., 2021). Given the contribution of RhopH2 and RhopH3 to NPP function, it is likely, therefore, that these two proteins play an essential accessory role.

There is also the possibility that CLAG3 is not the NPP channel and that other parasite or host proteins serve this function (Figure 2). Parasite-encoded proteins that exhibit homology to anion-selective channels have been identified among the Plasmodium transportome, including the outer membrane pore-forming protein (OMPP) found in bacteria and eukaryotic organelles such as the mitochondria and chloroplasts (Reddy and Saier, 2016) and the calcium-dependent, stress-gated ion channel (CSC) that belongs to the calciumdependent chloride channel (Ca-CIC) family (Martin, 2020). The localization of both of these proteins in Plasmodium has yet to be assessed; only CSC harbors a signal sequence, and neither protein is predicted to contain export motifs to facilitate trafficking into the erythrocyte after synthesis. Neither of these proteins interacts with members of the RhopH complex; thus, if they are involved in NPPs, they must function independently of the RhopH complex (Figure 2).

Immunoprecipitation experiments have revealed that $\sim 30$ parasite proteins affinity purify with RhopH2 (Counihan et al., 2017). While these proteins are exported or predicted to be exported into the host cytoplasm, none resemble channel proteins, and therefore, unlikely to serve as the NPP channel. Interestingly, erythrocyte anion exchanger 1 (AE1) affinity is purified with the RhopH complex, but as this endogenous protein is highly abundant, its binding may be non-specific. Moreover, if AE1 served as the NPP channel, it would have to be modified by the parasite during infection given that NPPs are not established until $15 \mathrm{~h}$ postinfection. While AE1 is progressively phosphorylated in response to oxidative stress when parasites transition from the ring to schizont stage (Pantaleo et al., 2010), the resulting reduction in AE1 affinity for the cytoskeleton and consequential membrane destabilization occur at the end of the cell cycle (Ferru et al., 2011), which is more consistent with a role in merozoite egress rather than NPP function.
Another erythrocyte voltage-dependent anion channel (VDAC), which is either expressed alone or as a component of the peripheral-type benzodiazepine receptor (PBR) complex, is the VDAC. In uninfected erythrocytes, PBR mediates the AE1-independent anion conductance and becomes upregulated when erythrocytes are infected by $P$. falciparum (Bouyer et al., 2011). The properties of VDAC resemble those of the NPP, and PBR ligands reduce membrane transport and conductance in $P$. falciparum-infected erythrocytes and block parasite growth in vitro. However, VDAC/PBR does not immunoprecipitate with the RhopH complex (Counihan et al., 2017), indicating that this transporter exists as an independent entity at the erythrocyte membrane and is not regulated by the RhopH components, but whether it associates with other parasite proteins is unknown. It also remains unknown what substrates VDAC/PBR transports in Plasmodium-infected erythrocytes. Thus, despite the importance of NPPs to parasite growth, the molecular composition of this channel still remains elusive.

\section{NUTRIENT TRANSFER ACROSS THE PVM}

The erythrocyte membrane is not the only barrier to host nutrient uptake by the parasite. Nutrients must also be able to cross the PVM. Soluble macromolecules such as amino acids and monosaccharides up to $1,400 \mathrm{Da}$ in size are able to readily cross this membrane via a channel that is permeable to both cations and anions (Desai and Rosenberg, 1997). Cell-attached patch clamping on parasites isolated from infected erythrocytes has revealed that this channel is present at the PVM in high density and is open most of the time (Desai et al., 1993). Permeation through this channel does not involve the binding of substrates; rather, the channel behaves like a sieve. Thus, transfer across the PVM is constrained by size of the solute and its diffusion coefficient in the cytosol (Desai et al., 1993; Desai and Rosenberg, 1997).

The PVM in the related apicomplexan parasite, Toxoplasma gondii, has a similar exclusion limit for macromolecules to that of Plasmodium (Schwab et al., 1994), suggesting that related channels may exist to transport soluble macromolecules across their respective PVMs. Two T. gondii proteins, termed GRA17 and GRA23, which are secreted from the dense granule organelles following invasion, perform this role. These proteins were shown to be required for the uptake of membrane-impermeable dyes lucifer yellow (522 Da) and the fluorescent form of 5-(and6)-carboxy-20,70-dichlorofluorescein diacetate (CDCFDA; 445 $\mathrm{Da}$ ) into the PV (Gold et al., 2015). In contrast, and consistent with the size constraint of this channel, dextran (3,000 Da) was excluded from entry. Moreover, injection of GRA17 and GRA23 cRNA into Xenopus oocytes altered their membrane conductance (Gold et al., 2015). Although the GRA proteins appear to act synergistically, it remains unclear if they have separate or overlapping functions. Studies in mice have revealed that GRA17-mediated PVM permeability is important for the growth of tachyzoites (the rapidly growing life stage of T. gondii) and virulence in mice, whereas both GRA17 and GRA23 are 
important for the viability of the $T$. gondii life stage inside cysts (Gold et al., 2015; Paredes-Santos et al., 2019; Li et al., 2020).

Interestingly, GRA17 and GRA23 show predicted structural similarity to Plasmodium EXP2, the pore-forming component of the Plasmodium translocon of exported proteins (PTEX) that is also secreted from dense granules. The PTEX machinery is responsible for the export of Plasmodium proteins into the host cytosol (de Koning-Ward et al., 2009), and GRA17 lossof-function phenotypes can be rescued by Plasmodium EXP2 (Gold et al., 2015). Yet, intriguingly, GRA17 or GRA23 does not play a role in protein export. However, recently, it was discovered that a PTEX-independent pool of EXP2 exists at the PVM in $P$. falciparum that facilitates the exchange of soluble macromolecules across this membrane (Garten et al., 2018). Indeed, there is a correlation between EXP2 expression levels and the frequency with which the channel can be detected. Removal of 53 amino acids from the $C$-terminus of EXP2, a region that is abundant in acidic residues, gives rise to a PVM channel with an altered voltage response. Combined, these studies suggest that EXP2 not only gates the PVM channel, but also forms the actual nutrient channel through the PVM. Interestingly, pores are also found in the PVM of the liver stage parasites (Bano et al., 2007). As EXP2 is also expressed in the liver stage parasites (Vaughan et al., 2012), it may be performing a similar role, pointing toward a conserved mechanism in both lifecycle stages.

If the nutrient pore of $P$. falciparum exists in an open confirmation, the incorporation of EXP2 into membranes would need to be regulated to permit nutrient exchange only at the PVM. One candidate regulatory protein is EXP1, a PVresident protein previously proposed to function as a glutathione S-transferase (Lisewski et al., 2014). EXP1 coimmunoprecipitates with EXP2 (Mesén-Ramírez et al., 2016) and is critical for PVM nutrient channel activity (Mesén-Ramírez et al., 2019). Conditional knockout of EXP1 using the dimerizable Cre system (Collins et al., 2013) led to slow growth phenotypes comparable to those resulting from amino acid starvation (Babbitt et al., 2012) or after conditional depletion of members of the RhopH complex (Counihan et al., 2017; Ito et al., 2017; Sherling et al., 2017). Compared with wild-type parasites, EXP1 knockout parasites showed a reduced frequency of channel detection by patch clamping, and transgenic parasites expressing limiting levels of EXP1 were hypersensitive to low levels of amino acids. These phenotypes may be attributable to altered PVM morphology and distribution of EXP2. EXP2 membrane association and protein export via PTEX did not appear to be affected (MesénRamírez et al., 2019), indicating that EXP1 does not regulate the insertion of EXP2 into the membrane. Nessel et al. (2020) have also generated EXP1 knockdown parasites using the TetRDOZI aptamer system (Ganesan et al., 2016) and similarly concluded that EXP1 is responsible for proper distribution of EXP2 at the PVM. However, as their knockout EXP1 parasites progressed further through the cell cycle than the EXP2 knockdown parasites, this led the authors to conclude that EXP1 does not contribute to EXP2-dependent transport activities. The differences between the findings of the two EXP1 studies may stem from the timing of EXP1 knockdown and when this impacts on PVM morphology. It is likely that disturbances to the PVM from EXP1 knockdown would also affect the localization/function of other PVM proteins that are critical for the parasite to complete the cell cycle, although this was not investigated.

RON3, a protein secreted from the rhoptry bulb and that localizes to the parasite periphery following invasion, has also been linked to PVM nutrient uptake. Parasites deficient in RON3 exhibit a similar phenotype to parasites deficient in EXP2 and fail to progress beyond the ring stages (Low et al., 2019). They are not only affected in their ability to import glucose, as they also fail to export proteins into the erythrocyte cytosol. However, RON3 is unlikely to be a direct interacting partner of nutrient pore EXP2 or PTEX EXP2; the BioID2 proximity ligase system fused to EXP2 did not reveal an interaction with RON3 (Mesén-Ramírez et al., 2016), and only few peptides were identified in a PTEX immunoprecipitation (de Koning-Ward et al., 2009), despite the fact that RON3 is a $263-\mathrm{kDa}$ protein. Thus, it remains unclear how RON3 contributes to both nutrient uptake and protein export, and further studies are required to assess whether EXP2 localization or assembly is affected in RON3-deficient parasites.

\section{TRANSPORT OF NUTRIENTS ACROSS THE PPM}

Once soluble macromolecules from the host have gained access to the PV, they can be then be transported across the PPM via an array of parasite transporters. A recent review by Martin (2020) has documented parasite transporters present at the different membranes in a parasitized erythrocyte, including the PPM, and so here we highlight only a subset for which there is experimental support for localization at the PPM. These include aquaglyceroporin (AQP), formate-lactate channel (FNT), putative copper channel, cationic amino acid transporter (NTP1), hexose transporter (HT1), putative amino acid transporter (AAT1, AAAP3), putative $\mathrm{Mg}^{2+}$ transporter, ATP: ADP antiporter (AAC1, AAC2), putative MCP1, folate-biopterin transporter (FT1, FT2), equilibrative nucleoside transporter (ENT, ENT4), $\mathrm{Zn}^{2+}$ and $\mathrm{Fe}^{2+}$ transporter (ZIPCO), the ABC transporters known as multidrug-resistant proteins (MDR1, MDR2, and MDR5) and multidrug-resistant associated proteins (MRP1 and MRP2), putative inorganic anion exchanger (SulP), Pi: $\mathrm{Na}^{+}$symporter (PiT), putative lipid/sterol: $\mathrm{H}^{+}$antiporter (NPC1R), V type $\mathrm{H}^{+}$ATPase, and a variety of P-ATPases (ATP1, ATP2, ATP4, ATP8, CuTP, and GCa).

Not all of the transporters at the PPM are essential for parasite survival. Gene disruption studies (reviewed in Martin, 2020) and a large-scale mutagenesis screen (Zhang et al., 2018) have provided insight into which of these many transporters are essential and therefore could constitute potential antimalaria drug targets. Although the identity of the PPM transporter for pantothenate remains unknown, it is also a candidate drug target given the reliance on pantothenate uptake for parasite survival. The putative pantothenate transporter (PPT) was suggested to serve this role as it could complement the growth phenotype of a yeast mutant deficient in pantothenate transport (Augagneur et al., 2013). However, a study using rodent malaria parasites 
revealed that this transporter, which is also referred to as the putative metabolite transporter TFP1, is not expressed at the PPM in asexual blood stages and instead localizes to osmiophilic bodies in gametocytes (Kehrer et al., 2016). Moreover, the $t f p 1$ gene is dispensable (Hart et al., 2014; Kehrer et al., 2016; Zhang et al., 2018). Alternate pantothenate transporter candidates have been proposed, including the putative metabolite/drug transporter umf and monocarboxylate transporter $m c p 1$ or one of the parasite's mitochondrial carrier superfamily proteins (Martin, 2020), but experimental evidence is lacking for any of these. Interestingly, the identity of the isoleucine PPM transporter, another potential antimalaria drug target due to the parasite's requirement to take up isoleucine, is also unknown. Proposed candidates include the putative amino acid transporters AAT1 and AAAP3 (Martin, 2020), both of which were not mutable in P. falciparum (Zhang et al., 2018).

\section{INGESTION OF HOST HEMOGLOBIN VIA FORMATION OF THE CYTOSTOME}

Unlike other microbes that source heme iron from hemoglobin through extraction from soluble and cell surface/wall receptors and subsequent transfer to cell wall and $\mathrm{ABC}$ transporters (Pishchany and Skaar, 2012), P. falciparum utilizes hemoglobin as a source of amino acids, which it instead acquires by endocytosing a large proportion of the host cell cytosol. This endocytosis process is non-selective, involving doublemembrane invagination of both the PVM and the PPM, resulting in formation of a cytostome and potentially a larger structure known as a phagotroph (Aikawa et al., 1966; Slomianny et al., 1985; Slomianny, 1990; Elliott et al., 2008). The content of cytostomes has been assumed to be subsequently transported to the parasite's digestive vacuole (DV); however, it is important to note that this has yet to be experimentally shown; consequently, the mechanism(s) by which hemoglobin and the host cell cytosol are delivered from endocytic structures to the DVs is yet to be resolved. We refer readers to a review by Spielmann et al. (2020) for proposed endocytic routes to the DV, which involve either the entire cytostome or only a portion pinching off and the subsequent transport to the DV, with potential involvement of intermediatory compartments. Digestion of hemoglobin occurs en route and within the DV (Abu Bakar et al., 2010), with the globin chains first cleaved from hemoglobin by aspartic proteases and cysteine endoproteases, and the liberated polypeptides then further digested by metalloproteases and series of aminopeptidases into oligopeptides and dipeptides to provide a source of amino acids for the parasite (Goldberg, 2005).

The molecular constituents that govern the uptake of the host cytosol by $P$. falciparum are also not resolved, largely because findings have either been conflicting or direct functional data have been lacking. We refer readers to two reviews that summarize the large body of work that has been undertaken to tease out potential molecular players in endocytosis of the host cell cytosol, with candidates including adapter protein-2 (AP-2), phosphatidylinositol-3-kinase, coronin, actin, dynamin, epidermal growth factor receptor substrate-15 (Eps15) homology domain (EHD) protein, soluble $N$-ethylmaleimide sensitive factor attachment protein (SNAP) receptor proteins (SNAREs), and Rab proteins (Spielmann et al., 2020; Xie et al., 2020). More recently, two studies from the Spielmann laboratory using genetic and proximity ligation approaches have shed further light on this process, revealing that components of a non-canonical endocytic apparatus are involved. In the first study, vacuolar protein sorting associated protein 4, which serves as an endolysosomal transport protein in other eukaryotes (Cowles et al., 1994; Piper et al., 1994), was shown to be required for host cell cytosol uptake (Jonscher et al., 2019). Then, a follow-up study examining the cellular function of Kelch 13 and its contribution to artemisinin resistance demonstrated that a Kelch-13 protein complex is also required for endocytosis of hemoglobin from the host cell (Birnbaum et al., 2020). This complex comprises Kelch 13, epidermal growth factor receptor substrate-15 (Eps15), ubiquitin carboxyl-terminal hydrolase (UBP1), AP-2, and a handful of other Kelch interacting proteins (Birnbaum et al., 2020). Unlike Kelch 13, which is only critical for the endocytosis of the host cell cytosol at ring stages, Eps15, UBP1, and AP-2 are required for endocytosis at both ring and trophozoite stages, such that inactivation of these proteins leads to a reduced transport of hemoglobin to the DV (Birnbaum et al., 2020). The identification of some of the molecular components of this endocytic pathway will now enable detailed mechanistic dissection of host cell cytosol uptake by the parasite.

\section{CONCLUDING REMARKS}

Until recently, many of the molecular players involved in nutrient uptake by Plasmodium parasites have remained elusive, largely due to the difficulties associated with genetic manipulation of the Plasmodium genome and studying the function of essential genes (de Koning-Ward et al., 2015). However, the use of advanced molecular approaches to conditionally knock out or knock down genes and their products has enabled a better understanding of this process. Many of the genes identified in the pathways discussed in this review are essential to parasite survival, which highlights that blocking parasite nutrient acquisition from the host with antimalaria drugs is a worthwhile strategy to pursue. For drugs such as artemisinin that are activated by hemoglobin-derived heme and thus are dependent on the adequate uptake of hemoglobin by the parasite, understanding how this is achieved could inform approaches that could be used to antagonize drug resistance. Conversely, the molecular constituents of the hemoglobin uptake pathway could also be targeted with drugs, albeit not in combination with artemisinin or other heme-activated compounds. However, there are still some key knowledge gaps remaining. Functional evidence for CLAG3 serving as the channel-forming component of the NPP is lacking; if it does form the channel, what processes occur to drive insertion of CLAG3 into the membrane. If CLAG3 does not perform this role, what protein does, and is it parasite-derived? What function is served by the other CLAG proteins; are they required for the uptake of specific substrates? Why is the RhopH 
complex synthesized at the end of the cell cycle and secreted during invasion when the NPPs are not established for $15 \mathrm{~h}$ later, and what essential role do RhopH2 and RhopH3 play in NPP formation? What process ensures that EXP2 can form both a protein export channel and a nutrient channel, and what regulates its assembly into the PVM so that it does not prematurely form pores in membranes prior to this time? And is EXP2 the only nutrient channel at the PVM? What is the identity of the PPM transporters that are responsible for the uptake of isoleucine and pantothenate that are known to be essential to the parasite? These are just some of the questions that remain unanswered and are key to fully understanding the essential process of nutrient acquisition by Plasmodium parasites.

\section{REFERENCES}

Abu Bakar, N., Klonis, N., Hanssen, E., Chan, C., and Tilley, L. (2010). Digestivevacuole genesis and endocytic processes in the early intraerythrocytic stages of Plasmodium falciparum. J. Cell Sci. 123, 441-450. doi: 10.1242/jcs.06 1499

Aikawa, M., Hepler, P. K., Huff, C. G., and Sprinz, H. (1966). The feeding mechanism of avian malarial parasites. J. Cell Biol. 28, 355-373. doi: 10.1083/ jcb.28.2.355

Augagneur, Y., Jaubert, L., Schiavoni, M., Pachikara, N., Garg, A., UsmaniBrown, S., et al. (2013). Identification and functional analysis of the primary pantothenate transporter, PfPAT, of the human malaria parasite Plasmodium falciparum. J. Biol. Chem. 288, 20558-20567. doi: 10.1074/jbc.m113.482992

Babbitt, S. E., Altenhofen, L., Cobbold, S. A., Istvan, E. S., Fennell, C., Doerig, C., et al. (2012). Plasmodium falciparum responds to amino acid starvation by entering into a hibernatory state. Proc. Natl. Acad. Sci. U S A. 109, E3278E3287.

Bano, N., Romano, J. D., Jayabalasingham, B., and Coppens, I. (2007). Cellular interactions of Plasmodium liver stage with its host mammalian cell. Int. J. Parasitol. 37, 1329-1341. doi: 10.1016/j.ijpara.2007.04.005

Birnbaum, J., Scharf, S., Schmidt, S., Jonscher, E., Hoeijmakers, W. A. M., Flemming, S., et al. (2020). A Kelch13-defined endocytosis pathway mediates artemisinin resistance in malaria parasites. Science 367, 51-59. doi: 10.1126/ science.aax 4735

Bouyer, G., Cueff, A., Egee, S., Kmiecik, J., Maksimova, Y., Glogowska, E., et al. (2011). Erythrocyte peripheral type benzodiazepine receptor/voltagedependent anion channels are upregulated by Plasmodium falciparum. Blood 118, 2305-2312. doi: 10.1182/blood-2011-01-329300

Chourasia, B. K., Deshmukh, A., Kaur, I., Paul, G., Panda, A., Rathore, S., et al. (2020). Plasmodium falciparum Clag9-Associated PfRhopH complex is involved in merozoite binding to human erythrocytes. Infect. Immun. 88, e00504-e00519.

Collins, C. R., Das, S., Wong, E. H., Andenmatten, N., Stallmach, R., Hackett, F., et al. (2013). Robust inducible Cre recombinase activity in the human malaria parasite Plasmodium falciparum enables efficient gene deletion within a single asexual erythrocytic growth cycle. Mol. Microbiol. 88, 687-701. doi: 10.1111/ mmi.12206

Comeaux, C. A., Coleman, B. I., Bei, A. K., Whitehurst, N., and Duraisingh, M. T. (2011). Functional analysis of epigenetic regulation of tandem RhopH1/clag genes reveals a role in Plasmodium falciparum growth. Mol. Microbiol. 80, 378-390. doi: 10.1111/j.1365-2958.2011.07572.x

Cooper, J. A., Ingram, L. T., Bushell, G. R., Fardoulys, C. A., Stenzel, D., Schofield, L., et al. (1988). The 140/130/105 kilodalton protein complex in the rhoptries of Plasmodium falciparum consists of discrete polypeptides. Mol. Biochem. Parasitol. 29, 251-260. doi: 10.1016/0166-6851(88) 90080-1

Cortés, A., Carret, C., Kaneko, O., Yim Lim, B. Y., Ivens, A., and Holder, A. A. (2007). Epigenetic silencing of Plasmodium falciparum genes linked to erythrocyte invasion. PLoS Pathog 3:e107. doi: 10.1371/journal.ppat.0030107

\section{AUTHOR CONTRIBUTIONS}

NC, JM, and TdK-W were responsible for writing and editing manuscript drafts and the final document. All authors contributed to the article and approved the submitted version.

\section{FUNDING}

This work was supported by NHMRC grant 1182000. TdK-W acknowledges support of an NHMRC Senior Research Fellowship (1136300).

Counihan, N. A., Chisholm, S. A., Bullen, H. E., Srivastava, A., Sanders, P. R., Jonsdottir, T. K., et al. (2017). Plasmodium falciparum parasites deploy RhopH2 into the host erythrocyte to obtain nutrients, grow and replicate. eLife 6:e23217.

Counihan, N. A., Kalanon, M., Coppel, R. L., and De Koning-Ward, T. F. (2013). Plasmodium rhoptry proteins: why order is important. Trends Parasitol. 29, 228-236. doi: 10.1016/j.pt.2013.03.003

Cowles, C. R., Emr, S. D., and Horazdovsky, B. F. (1994). Mutations in the VPS45 gene, a SEC1 homologue, result in vacuolar protein sorting defects and accumulation of membrane vesicles. J. Cell Sci. 107(Pt 12), 3449-3459.

Cowman, A. F., Baldi, D. L., Healer, J., Mills, K. E., O’donnell, R. A., Reed, M. B., et al. (2000). Functional analysis of proteins involved in Plasmodium falciparum merozoite invasion of red blood cells. FEBS Lett. 476, 84-88.

de Koning-Ward, T. F., Gilson, P. R., Boddey, J. A., Rug, M., Smith, B. J., Papenfuss, A. T., et al. (2009). A newly discovered protein export machine in malaria parasites. Nature 459, 945-949. doi: 10.1038/nature08104

de Koning-Ward, T. F., Gilson, P. R., and Crabb, B. S. (2015). Advances in molecular genetic systems in malaria. Nat. Rev. Microbiol. 13, 373-387. doi: $10.1038 /$ nrmicro3450

Desai, S. A. (2012). Ion and nutrient uptake by malaria parasite-infected erythrocytes. Cell Microbiol. 14, 1003-1009. doi: 10.1111/j.1462-5822.2012. 01790.x

Desai, S. A., Krogstad, D. J., and McCleskey, E. W. (1993). A nutrient-permeable channel on the intraerythrocytic malaria parasite. Nature 362, 643-646. doi: $10.1038 / 362643 \mathrm{a} 0$

Desai, S. A., and Rosenberg, R. L. (1997). Pore size of the malaria parasite's nutrient channel. Proc. Natl. Acad. Sci. U S A. 94, 2045-2049. doi: 10.1073/pnas.94.5. 2045

Divo, A. A., Geary, T. G., Davis, N. L., and Jensen, J. B. (1985). Nutritional requirements of Plasmodium falciparum in culture. I. exogenously supplied dialyzable components necessary for continuous growth. J. Protozool. 32, 59-64. doi: 10.1111/j.1550-7408.1985.tb03013.x

Downie, M. J., Kirk, K., and Mamoun, C. B. (2008). Purine salvage pathways in the intraerythrocytic malaria parasite Plasmodium falciparum. Eukaryot Cell 7, 1231-1237. doi: 10.1128/ec.00159-08

Elliott, D. A., Mcintosh, M. T., Hosgood, H. D. III, Chen, S., Zhang, G., Baevova, P., et al. (2008). Four distinct pathways of hemoglobin uptake in the malaria parasite Plasmodium falciparum. Proc. Natl. Acad. Sci. U S A. 105, 2463-2468. doi: 10.1073/pnas.0711067105

Ferru, E., Giger, K., Pantaleo, A., Campanella, E., Grey, J., Ritchie, K., et al. (2011). Regulation of membrane-cytoskeletal interactions by tyrosine phosphorylation of erythrocyte band 3. Blood 117, 5998-6006. doi: 10.1182/blood-2010-11317024

Ganesan, S. M., Falla, A., Goldfless, S. J., Nasamu, A. S., and Niles, J. C. (2016). Synthetic RNA-protein modules integrated with native translation mechanisms to control gene expression in malaria parasites. Nat. Commun. 7:10727.

Garten, M., Nasamu, A. S., Niles, J. C., Zimmerberg, J., Goldberg, D. E., and Beck, J. R. (2018). EXP2 is a nutrient-permeable channel in the vacuolar membrane of Plasmodium and is essential for protein export via PTEX. Nat. Microbiol. 3, 1090-1098. doi: 10.1038/s41564-018-0222-7 
Ginsburg, H., Kutner, S., Krugliak, M., and Cabantchik, Z. I. (1985). Characterization of permeation pathways appearing in the host membrane of Plasmodium falciparum infected red blood cells. Mol. Biochem. Parasitol. 14, 313-322. doi: 10.1016/0166-6851(85)90059-3

Goel, S., Valiyaveettil, M., Achur, R. N., Goyal, A., Mattei, D., Salanti, A., et al. (2010). Dual stage synthesis and crucial role of cytoadherence-linked asexual gene 9 in the surface expression of malaria parasite var proteins. Proc. Natl. Acad. Sci. U S A. 107, 16643-16648. doi: 10.1073/pnas. 1002568107

Gold, D. A., Kaplan, A. D., Lis, A., Bett, G. C., Rosowski, E. E., Cirelli, K. M., et al. (2015). The Toxoplasma dense granule proteins GRA17 and GRA23 mediate the movement of small molecules between the host and the parasitophorous vacuole. Cell Host Microbe 17, 642-652. doi: 10.1016/j.chom.2015.04.003

Goldberg, D. E. (2005). Hemoglobin degradation. Curr. Top Microbiol. Immunol. 295, 275-291.

Gulati, S., Ekland, E. H., Ruggles, K. V., Chan, R. B., Jayabalasingham, B., Zhou, B., et al. (2015). Profiling the essential nature of lipid metabolism in asexual blood and gametocyte stages of Plasmodium falciparum. Cell Host Microbe 18, 371-381. doi: 10.1016/j.chom.2015.08.003

Gupta, A., Bokhari, A. A. B., Pillai, A. D., Crater, A. K., Gezelle, J., Saggu, G., et al. (2020). Complex nutrient channel phenotypes despite Mendelian inheritance in a Plasmodium falciparum genetic cross. PLoS Pathog 16:e1008363. doi: 10.1371/ journal.ppat.1008363

Hart, R. J., Lawres, L., Fritzen, E., Ben Mamoun, C., and Aly, A. S. (2014). Plasmodium yoelii vitamin B5 pantothenate transporter candidate is essential for parasite transmission to the mosquito. Sci. Rep. 4:5665.

Ito, D., Schureck, M. A., and Desai, S. A. (2017). An essential dual-function complex mediates erythrocyte invasion and channel-mediated nutrient uptake in malaria parasites. eLife 6:e23485.

Janse, C. J., Kroeze, H., Van Wigcheren, A., Mededovic, S., Fonager, J., FrankeFayard, B., et al. (2011). A genotype and phenotype database of genetically modified malaria-parasites. Trends Parasitol. 27, 31-39. doi: 10.1016/j.pt.2010. 06.016

Jonscher, E., Flemming, S., Schmitt, M., Sabitzki, R., Reichard, N., Birnbaum, J., et al. (2019). PfVPS45 is required for host cell cytosol uptake by malaria blood stage parasites. Cell Host Microbe 25, 166-173.e5.

Kaneko, O., Tsuboi, T., Ling, I. T., Howell, S., Shirano, M., Tachibana, M., et al. (2001). The high molecular mass rhoptry protein, RhopH1, is encoded by members of the clag multigene family in Plasmodium falciparum and Plasmodium yoelii. Mol. Biochem. Parasitol. 118, 223-231. doi: 10.1016/s01666851(01)00391-7

Kaneko, O., Yim Lim, B. Y., Iriko, H., Ling, I. T., Otsuki, H., Grainger, M., et al. (2005). Apical expression of three RhopH1/Clag proteins as components of the Plasmodium falciparum RhopH complex. Mol. Biochem. Parasitol. 143, 20-28. doi: 10.1016/j.molbiopara.2005.05.003

Kehrer, J., Singer, M., Lemgruber, L., Silva, P. A., Frischknecht, F., and Mair, G. R. (2016). A putative small solute transporter is responsible for the secretion of G377 and TRAP-containing secretory vesicles during Plasmodium gamete egress and sporozoite motility. PLoS Pathog 12:e1005734. doi: 10.1371/journal. ppat. 1005734

Kirk, K., Horner, H. A., Elford, B. C., Ellory, J. C., and Newbold, C. I. (1994). Transport of diverse substrates into malaria-infected erythrocytes via a pathway showing functional characteristics of a chloride channel. J. Biol. Chem. 269, 3339-3347. doi: 10.1016/s0021-9258(17)41868-0

Li, T. T., Wang, J. L., Liang, Q. L., Sun, L. X., Zhang, H. S., Zhang, Z. W., et al. (2020). Effect of deletion of gra17 and gra23 genes on the growth, virulence, and immunogenicity of type II Toxoplasma gondii. Parasitol. Res. 119, 2907-2916. doi: 10.1007/s00436-020-06815-z

Ling, I. T., Florens, L., Dluzewski, A. R., Kaneko, O., Grainger, M., Yim Lim, B. Y., et al. (2004). The Plasmodium falciparum clag9 gene encodes a rhoptry protein that is transferred to the host erythrocyte upon invasion. Mol. Microbiol. 52, 107-118. doi: 10.1111/j.1365-2958.2003.03969.x

Lisewski, A. M., Quiros, J. P., Ng, C. L., Adikesavan, A. K., Miura, K., Putluri, N., et al. (2014). Supergenomic network compression and the discovery of EXP1 as a glutathione transferase inhibited by artesunate. Cell 158, 916-928. doi: 10.1016/j.cell.2014.07.011

Liu, J., Istvan, E. S., Gluzman, I. Y., Gross, J., and Goldberg, D. E. (2006). Plasmodium falciparum ensures its amino acid supply with multiple acquisition pathways and redundant proteolytic enzyme systems. Proc. Natl. Acad. Sci. U S A. 103, 8840-8845. doi: 10.1073/pnas.0601876103

Low, L. M., Azasi, Y., Sherling, E. S., Garten, M., Zimmerberg, J., Tsuboi, T., et al. (2019). Deletion of Plasmodium falciparum protein RON3 affects the functional translocation of exported proteins and glucose uptake. mBio 10:e01460-19.

Martin, R. E. (2020). The transportome of the malaria parasite. Biol. Rev. Camb. Philos. Soc. 95, 305-332. doi: 10.1111/brv.12565

Martin, R. E., and Kirk, K. (2007). Transport of the essential nutrient isoleucine in human erythrocytes infected with the malaria parasite Plasmodium falciparum. Blood 109, 2217-2224. doi: 10.1182/blood-2005-11-026963

Mesén-Ramírez, P., Bergmann, B., Tran, T. T., Garten, M., Stäcker, J., Naranjo-Prado, I., et al. (2019). EXP1 is critical for nutrient uptake across the parasitophorous vacuole membrane of malaria parasites. PLoS Biol. 17:e3000473. doi: 10.1371/journal.pbio.3000473

Mesén-Ramírez, P., Reinsch, F., Blancke Soares, A., Bergmann, B., Ullrich, A. K., Tenzer, S., et al. (2016). Stable translocation intermediates jam global protein export in Plasmodium falciparum parasites and link the PTEX component EXP2 with translocation activity. PLoS Pathog 12:e1005618. doi: 10.1371/journal.ppat. 1005618

Mira-Martinez, S., Rovira-Graells, N., Crowley, V. M., Altenhofen, L. M., Llinas, M., and Cortes, A. (2013). Epigenetic switches in clag3 genes mediate blasticidin S resistance in malaria parasites. Cell Microbiol. 15, 1913-1923.

Nacer, A., Roux, E., Pomel, S., Scheidig-Benatar, C., Sakamoto, H., Lafont, F., et al. (2011). clag9 is not essential for PfEMP1 surface expression in noncytoadherent Plasmodium falciparum parasites with a chromosome 9 deletion. PLoS One 6:e29039. doi: 10.1371/journal.pone.0029039

Nessel, T., Beck, J. M., Rayatpisheh, S., Jami-Alahmadi, Y., Wohlschlegel, J. A., Goldberg, D. E., et al. (2020). EXP1 is required for organisation of EXP2 in the intraerythrocytic malaria parasite vacuole. Cell Microbiol. 22:e13168.

Nguitragool, W., Bokhari, A. A., Pillai, A. D., Rayavara, K., Sharma, P., Turpin, B., et al. (2011). Malaria parasite clag3 genes determine channel-mediated nutrient uptake by infected red blood cells. Cell 145, 665-677. doi: 10.1016/j.cell.2011. 05.002

Nguitragool, W., Rayavara, K., and Desai, S. A. (2014). Proteolysis at a specific extracellular residue implicates integral membrane CLAG3 in malaria parasite nutrient channels. PLoS One 9:e93759. doi: 10.1371/journal.pone.0093759

O’Neal, A. J., Butler, L. R., Rolandelli, A., Gilk, S. D., and Pedra, J. H. (2020). Lipid hijacking: a unifying theme in vector-borne diseases. eLife 9:e61675.

Overman, R. R. (1947). Reversible permeability alterations in the erythrocytes of the malarious monkey. Fed. Proc. 6:174.

Pantaleo, A., De Franceschi, L., Ferru, E., Vono, R., and Turrini, F. (2010). Current knowledge about the functional roles of phosphorylative changes of membrane proteins in normal and diseased red cells. J. Proteomics 73, 445-455. doi: 10.1016/j.jprot.2009.08.011

Paredes-Santos, T., Wang, Y., Waldman, B., Lourido, S., and Saeij, J. P. (2019). The GRA17 parasitophorous vacuole membrane permeability pore contributes to bradyzoite viability. Front. Cell Infect. Microbiol. 9:321. doi: 10.3389/fcimb. 2019.00321

Pillai, A. D., Nguitragool, W., Lyko, B., Dolinta, K., Butler, M. M., Nguyen, S. T., et al. (2012). Solute restriction reveals an essential role for clag3-associated channels in malaria parasite nutrient acquisition. Mol. Pharmacol. 82, 11041114. doi: $10.1124 / \mathrm{mol} .112 .081224$

Piper, R. C., Whitters, E. A., and Stevens, T. H. (1994). Yeast Vps45p is a Seclp-like protein required for the consumption of vacuole-targeted, post-Golgi transport vesicles. Eur. J. Cell Biol. 65, 305-318.

Pishchany, G., and Skaar, E. P. (2012). Taste for blood: hemoglobin as a nutrient source for pathogens. PLoS Pathog 8:e1002535. doi: 10.1371/journal.ppat. 1002535

Pretini, V., Koenen, M. H., Kaestner, L., Fens, M., Schiffelers, R. M., Bartels, M., et al. (2019). Red blood cells: chasing interactions. Front. Physiol. 10:945. doi: 10.3389/fphys.2019.00945

Reddy, B. L., and Saier, M. H., Jr. (2016). Properties andphylogeny of 76 families of bacterial and eukaryotic organellar outer membrane pore-forming proteins. PLoS One 11:e0152733. doi: 10.1371/journal.pone.0152733

Reyes, P., Rathod, P. K., Sanchez, D. J., Mrema, J. E., Rieckmann, K. H., and Heidrich, H. G. (1982). Enzymes of purine and pyrimidine metabolism from 
the human malaria parasite. Plasmodium falciparum. Mol. Biochem. Parasitol. 5, 275-290. doi: 10.1016/0166-6851(82)90035-4

Saliba, K. J., Horner, H. A., and Kirk, K. (1998). Transport and metabolism of the essential vitamin pantothenic acid in human erythrocytes infected with the malaria parasite Plasmodium falciparum. J. Biol. Chem. 273, 10190-10195. doi: 10.1074/jbc.273.17.10190

Sam-Yellowe, T. Y., Shio, H., and Perkins, M. E. (1988). Secretion of Plasmodium falciparum rhoptry protein into the plasma membrane of host erythrocytes. J. Cell Biol. 106, 1507-1513. doi: 10.1083/jcb.106.5.1507

Schureck, M. A., Darling, J. E., Merk, A., Shao, J., Daggupati, G., Srinivasan, P., et al. (2021). Malaria parasites use a soluble RhopH complex for erythrocyte invasion and an integral form for nutrient uptake. eLife 10:e65282.

Schwab, J. C., Beckers, C. J., and Joiner, K. A. (1994). The parasitophorous vacuole membrane surrounding intracellular Toxoplasma gondii functions as a molecular sieve. Proc. Natl. Acad. Sci. U S A. 91, 509-513. doi: 10.1073/pnas. 91.2.509

Sharma, P., Rayavara, K., Ito, D., Basore, K., and Desai, S. A. (2015). A CLAG3 mutation in an amphipathic transmembrane domain alters malaria parasite nutrient channels and confers leupeptin resistance. Infect. Immun. 83, 25662574. doi: 10.1128/iai.02966-14

Sharma, P., Wollenberg, K., Sellers, M., Zainabadi, K., Galinsky, K., Moss, E., et al. (2013). An epigenetic antimalarial resistance mechanism involving parasite genes linked to nutrient uptake. J. Biol. Chem. 288, 19429-19440. doi: 10.1074/ jbc.m113.468371

Sherling, E. S., Knuepfer, E., Brzostowski, J. A., Miller, L. H., Blackman, M. J., and Van Ooij, C. (2017). The Plasmodium falciparum rhoptry protein RhopH3 plays essential roles in host cell invasion and nutrient uptake. eLife 6:e23239.

Sherman, I. W. (1977). Transport of amino acids and nucleic acid precursors in malarial parasites. Bull. World Health Organ. 55, 211-225.

Slomianny, C. (1990). Three-dimensional reconstruction of the feeding process of the malaria parasite. Blood Cells 16, 369-378.

Slomianny, C., Prensier, G., and Charet, P. (1985). Ingestion of erythrocytic stroma by Plasmodium chabaudi trophozoites: ultrastructural study by serial sectioning and 3-dimensional reconstruction. Parasitology 90(Pt 3), 579-588. doi: 10. 1017/s0031182000055578

Spielmann, T., Gras, S., Sabitzki, R., and Meissner, M. (2020). Endocytosis in Plasmodium and toxoplasma parasites. Trends Parasitol. 36, 520-532. doi: 10.1016/j.pt.2020.03.010

Spry, C., Van Schalkwyk, D. A., Strauss, E., and Saliba, K. J. (2010). Pantothenate utilization by Plasmodium as a target for antimalarial chemotherapy. Infect. Disord. Drug Targets 10, 200-216. doi: 10.2174/187152610791163390
Staines, H. M., Ellory, J. C., and Kirk, K. (2001). Perturbation of the pump-leak balance for $\mathrm{Na}(+)$ and $\mathrm{K}(+)$ in malaria-infected erythrocytes. Am. J. Physiol. Cell Physiol. 280, C1576-C1587.

Trenholme, K. R., Gardiner, D. L., Holt, D. C., Thomas, E. A., Cowman, A. F., and Kemp, D. J. (2000). clag9: a cytoadherence gene in Plasmodium falciparum essential for binding of parasitized erythrocytes to CD36. Proc. Natl. Acad. Sci. U S A. 97, 4029-4033. doi: 10.1073/pnas.040561197

Vaughan, A. M., Mikolajczak, S. A., Wilson, E. M., Grompe, M., Kaushansky, A., Camargo, N., et al. (2012). Complete Plasmodium falciparum liver-stage development in liver-chimeric mice. J. Clin. Invest. 122, 3618-3628. doi: 10. $1172 /$ jci62684

Vincensini, L., Fall, G., Berry, L., Blisnick, T., and Braun Breton, C. (2008). The RhopH complex is transferred to the host cell cytoplasm following red blood cell invasion by Plasmodium falciparum. Mol. Biochem. Parasitol. 160, 81-89. doi: 10.1016/j.molbiopara.2008.04.002

Wang, P., Wang, Q., Sims, P. F., and Hyde, J. E. (2007). Characterisation of exogenous folate transport in Plasmodium falciparum. Mol. Biochem. .Parasitol. 154, 40-51. doi: 10.1016/j.molbiopara.2007.04.002

World Health Organization (2019). World Malaria Report 2019. Geneva: World Health Organization.

Wrenger, C., Eschbach, M. L., Müller, I. B., Laun, N. P., Begley, T. P., and Walter, R. D. (2006). Vitamin B1 de novo synthesis in the human malaria parasite Plasmodium falciparum depends on external provision of 4-amino-5hydroxymethyl-2-methylpyrimidine. Biol. Chem. 387, 41-51.

Xie, S. C., Ralph, S. A., and Tilley, L. (2020). K13, the cytostome, and artemisinin resistance. Trends Parasitol. 36, 533-544. doi: 10.1016/j.pt.2020.03.006

Zhang, M., Wang, C., Otto, T. D., Oberstaller, J., Liao, X., Adapa, S. R., et al. (2018). Uncovering the essential genes of the human malaria parasite Plasmodium falciparum by saturation mutagenesis. Science 360:eaa7847.

Zhang, Y. (2008). I-TASSER server for protein 3D structure prediction. BMC Bioinform. 9:40. doi: 10.1186/1471-2105-9-40

Conflict of Interest: The authors declare that the research was conducted in the absence of any commercial or financial relationships that could be construed as a potential conflict of interest.

Copyright (c) 2021 Counihan, Modak and de Koning-Ward. This is an open-access article distributed under the terms of the Creative Commons Attribution License (CC BY). The use, distribution or reproduction in other forums is permitted, provided the original author(s) and the copyright owner(s) are credited and that the original publication in this journal is cited, in accordance with accepted academic practice. No use, distribution or reproduction is permitted which does not comply with these terms. 